\title{
La psicomotricidad como alternativa de reivindicación de la importancia del cuerpo y el movimiento para el desarrollo humano
}

\section{Psychomotor function as an alternative to vindicate the importance of body and motion in human development}

\author{
Álvaro José Gracia Díaz, Ph.D*
}

\section{Resumen}

\begin{abstract}
El movimiento es una necesidad básica de la vida y, por ende, del desarrollo humano. Esta condición se ha ido insertando profundamente en la naturaleza de la humanidad desde tiempos inmemoriales, durante los cuales operaron los procesos para convertir al ser humano en un ser en movimiento, que ha trascendido sus límites biológicos y cuya condición ha perdurado durante los últimos miles de años.

Hoy en día se percibe que el objetivo de las corrientes de educación tradicional se centra en la preparación de ciudadanos laboralmente capaces, efectivos y funcionales, en un mundo que maneja un ritmo de vida demasiado acelerado y donde, en consecuencia, no hay tiempo para detenerse a sentir el cuerpo, sus achaques y reclamos debidos al afán y el olvido hacia él. Por esto, se propone una intervención educativa, la psicomotricidad, en la que se rescate el verdadero sentido de lo humano en lo referente a su capacidad de imaginar, crear y sentir por medio del cuerpo y el intelecto.
\end{abstract}

Palabras clave: Psicomotricidad, movimiento, cuerpo, desarrollo, pedagogía tradicional.

\section{Abstract}

Motion is a basic need in life and, therefore, in human development. It has been a part of the nature of humankind for thousands of years. During this time, the processes by which the human being turned into an in-motion creature who has gone beyond biological boundaries have taken place.

Today, it is generally accepted that traditional pedagogy trends aim at preparing citizens as skillful, effective and functional workers, who know how to manage themselves in a fast-paced world. However, this frenetic lifestyle leaves people with no time to stop and feel their body, its ailments and claims about rush and carelessness towards it. For this reason, there is a need to propose an educational intervention plan, i. e. the psychomotor function, in which the real sense of human aspects associated with the capability to imagine, create and feel through one's body and intellect is vindicated.

Key Words: $\quad$ Psychomotor function, motion, body, human development, traditional pedagogy.

Fecha de recepción: 20 de septiembre de 2006

Fecha de aceptación: 12 de diciembre de 2006

* Profesor de la Universidad Pedagógica Nacional. 


\section{De ser biológico a sujeto cultural autónomo}

Desde el inicio del proceso evolutivo humano el sistema motor ha estado presente, como sigue estándolo en la actualidad, y ha sido el que ha motivado el proceso en una reacción en cadena que aún hoy continúa y que puede afirmarse proseguirá, siempre y cuando la especie humana tome conciencia de su condición terrenaluniversal y redireccione su existencia, estableciendo condiciones naturales y socioculturales que le permitan continuar sobreviviendo como especie.

Ninguno de los sistemas del cuerpo humano (digestivo, excretor, respiratorio, circulatorio) está directamente bajo el control voluntario de la mente, aunque puedan verse afectados por las emociones y las tensiones psicológicas. Por tanto, la única forma que tienen de desarrollarse y fortalecerse, así como de mantenerse, es mediante el efecto del ejercicio de los músculos esqueléticos voluntarios y, en especial, de los grandes músculos y músculos fundamentales del tronco y porción superior de piernas y brazos (Barrow y Brown, 1992).

Así, el movimiento es una necesidad básica de la vida $y$, por ende, del desarrollo humano. Esta condición se ha insertado profundamente en la naturaleza de la humanidad desde tiempos inmemoriales, durante los cuales operaron los procesos para convertir al ser humano en un ser activo en movimiento, que ha trascendido sus límites biológicos y cuya condición ha perdurado durante los últimos miles de años.

Como lo afirma Hugo Zemelman (2004-2005), antiguamente se consideraba que el cuerpo era el habitáculo del alma y su formación y desarrollo debía estar encaminada al cuidado de ésta. Durante siglos, se torturó al cuerpo negando sus sentidos y capacidades para que no expresara afecciones grotescas o soeces, que no estuvieran de acuerdo con la idiosincrasia de la época. En Oriente, los antiguos monjes soportaban extensas jornadas de inanición para que el cuerpo y sus necesidades orgánicas no interfirieran los procesos de meditación y búsqueda espiritual.

Hoy en día se percibe que el objetivo de las corrientes de educación tradicional se centra en la preparación de ciudadanos laboralmente capaces, efectivos y funcionales, en un mundo que maneja un ritmo de vida demasiado acelerado y donde, en consecuencia, no hay tiempo para detenerse a sentir el cuerpo, sus achaques y reclamos debidos al afán y el olvido hacia él. Por eso, se hace necesaria una intervención educativa en la que se rescate el verdadero sentido de lo humano en lo referen- te a su capacidad de imaginar, crear y sentir por medio del cuerpo y el intelecto.

Es fundamental, en el ámbito escolar, reconocer y entender el cuerpo y el cerebro como una unidad de acción y, en consecuencia, los estudiantes deben ser educados como conjunto. Las ciencias gozan de un espacio privilegiado en los programas educativos, mientras las artes apenas si aparecen parcialmente. Se ha olvidado la gran dimensión de la facultad de expresión y creación, sistematizando el cuerpo en ecuaciones y laboratorios. No obstante, con esto no se tiene la intención de desvalorizar la ciencia, sino de hacer ver que ésta ha desplazado de manera notable las artes humanas y sociales.

Con dificultad, algunos educadores, como adecuadamente lo plantea Francisco Cajiao (1996), comprenden que no hay justificación para imponer la quietud y el silencio en las aulas en busca de una mayor concentración y eficacia en la asimilación de los contenidos escolares. El cuerpo es intelecto y la imaginación es la ventana hacia la ciencia; somos cuerpo y por eso tenemos que empezar por el cuerpo una educación hacia y a través de él, aun cuando ello parezca salirse de los cánones de la enseñanza actual, pero generando nuevas experiencias, nuevos aprendizajes y nuevos modelos de desarrollo. La psicomotricidad enfoca la educación hacia una corriente que eduque y empiece desde el cuerpo, enseñándole al niño a valerse y a expresarse por su propio movimiento e interés.

El cuerpo es el punto de partida de la maduración del individuo y es donde se inscribirán las vivencias positivas y negativas que enfrentará durante de su historia. $L a$ psicomotricidad retoma los valores marginados, presentes en el movimiento humano y reconoce sus funciones comunicativas, afectivas y fisiológicas. La psicomotricidad es una cualidad única en la especie humana; integración perfecta entre lo evidente, lo físico y lo intangible, que corresponde a la psique.

La base biológica de la vida humana se manifiesta de forma más notoria en el sistema nervioso. El movimiento no acontece en el vacío, sino que debe ser iniciado por un estímulo de algún tipo o por los procesos de pensamiento. Los centros nerviosos se han fortalecido mediante la acción de músculos, de tal forma que, en el proceso evolutivo, se desarrollaron grupos musculares fundamentales, encargados de la postura y el transporte, denominados patrones generales de movimiento (Barrow y Brown, 1992). Estos músculos, desde la perspectiva filogenética, son los más antiguos y más resistentes, y sus movimientos se conocen como acti- 
vidad de la musculatura mayor. Esto significa que las adaptaciones posturales constituyen la base de todos los movimientos y, junto con las de transporte, establecen los patrones de movimiento para afrontar la fuerza de gravedad.

De igual manera, los patrones especiales de movimiento o de manipulación se expresan en la acción de la musculatura menor que controla las manos y otros movimientos periféricos (Barrow y Brown, 1992). En un contexto filogenético, estos músculos más pequeños, que involucran los mecanismos de las manos y del habla, se conocen como músculos accesorios. Aparecen más tarde en el ciclo evolutivo, por lo que son más jóvenes, más complejos y menos eficientes para desarrollar la resistencia y la estabilidad del sistema nervioso que el grupo fundamental más antiguo. Así, a partir de estas ideas puede afirmarse que el desarrollo y el aprendizaje humanos se manifiestan con mucha decisión, a medida que se utilizan movimientos para controlar y manipular objetos y materiales en el espacio.

El desarrollo de los estudios genéticos revela que la corteza cerebral comenzó a aumentar en complejidad cuando entraron en acción las coordinaciones finas de las manos. Este hecho consolida la importancia de la postura y la posición bípedas, que dejaron libres las manos para la manipulación. Puede inferirse que todo el comportamiento intelectual del desarrollo, el control y la coordinación se debe primero a los músculos fundamentales del organismo y más tarde a los músculos accesorios. Por tanto, el desarrollo intelectual ha de ir precedido por el movimiento, y por un movimiento de grupos musculares en destrezas fundamentales y especiales.

En este orden de ideas, los delicados patrones de movimiento, coordinados con las manos, combinados por los mecanismos de la participación visual, fueron movimientos de un orden superior que, "al final" del proceso evolutivo, dieron lugar a características de gran precisión, como el habla e indirectamente las operaciones simbólicas de lectura y escritura. Este movimiento, en una relación especial con las experiencias perceptivomotoras de instante y las almacenadas, condujo a la formación de los conceptos, los cuales, a su vez, abrieron las puertas para el proceso de pensamiento.

Con el habla y el lenguaje se posibilitó la acumulación de cultura, lo que contribuyó al desarrollo de capacidades humanas como el pensamiento, la memoria, la imaginación, la creación, la inventiva y toda la subjetividad y multidimensionalidad humana. La evolución biológica humana alcanzó un punto desde el cual pudo iniciarse la evolución la cultural. A partir de ese momento interactuaron conjuntamente la evolución biológica y cultural, lo que dio como resultado la aparición de seres humanos. Estudios antropológicos ponen de manifiesto que el homo sapiens se ha desarrollado durante innumerables años hasta convertirse en un ser muy inteligente.

El desarrollo de la llamada materia gris o neocortex hace precisamente lo que es humano, en el orden del conocimiento, con su capacidad de autoconciencia, de imaginación, de anticipación, de iniciativa, de encuentro entre estímulo y respuesta, de lenguaje, de cálculo, de pensamiento, de sentido de justicia, con el poder de fijarse propósitos libremente y con la apertura a deseos ilimitados, a la necesidad de placeres estéticos, a bienes abstractos y al anhelo de explorar lo insondable e infinito.

Ommo Grupe (1976) plantea que Herder "definió al hombre como el primer liberado de la creación", cuya nota característica está "en poder elegir aun cuando elija lo peor"; pero a la vez, y como consecuencia, "el hombre se convierte en su propio fin y meta de su elaboración". Así, con la vida del hombre comienza también la educación; venido al mundo con fuerzas y miembros, tiene que aprender su empleo, aplicación y desarrollo. De igual manera, Kant plantea que, a diferencia del animal que ha de obedecer a sus instintos, el hombre tiene que elaborar él mismo el plan de su comportamiento. Y lo mismo piensa Schiller, cuando afirma que el hombre posee la capacidad de intervenir como persona "en el currículo de la necesidad" y desarrollar, por sí mismo, el destino que le ha asignado la naturaleza, y "que la razón de que el hombre sea un ser abierto al mundo está en su liberación existencial de lo orgánico". Buytendijk (citado en Grupe, 1976) hace lo propio al asegurar que es:

el proceso de humanización de la persona humana responsable de lo auténtico y propiamente humano del movimiento del hombre, movimiento que, por tanto, no corresponde a la simple estructura vectorial del campo, sino que es elegido y querido gracias a que el hombre no está forzado por la situación.

\section{La Educación Física como disciplina y su objeto de estudio}

Como el hombre pasó de un ser biológico a un sujeto cultural autónomo,

hay que pensar a la educación física más allá de la simple generación de destrezas deportivas para po- 
nerla en escenarios eminentemente competitivos y más bien comprender que desde la acción motriz se estructura toda la realidad multidimensional y unitaria de lo humano (Pachón, 2005).

La primera reflexión sobre la educación física se particulariza en su objeto de estudio propio, que se centra en la acción motriz. En éste se integran el sujeto, la acción misma y el contexto (condiciones externas) de la acción, para conformar un cuerpo conceptual específico. Se trata de otorgar a la acción motriz el carácter forjador de lo humano, o a la motricidad, la cual "excede el simple proceso espacio temporal para situarse en un proceso de complejidad humana: cultural, simbólico, social, político, afectivo, intelectual... y por supuesto motor" (Trigo, 2000); es decir, multidimensional. En esta línea de pensamiento se expresa José María Cagigal (citado en Trigo, 2000), al afirmar que "el movimiento es una de las primeras providencias antropológicas del ser humano", en la medida que lo designa como el instrumento antropológico natural de la educación física.

Desde esta perspectiva, se intenta superar el reduccionismo en que ha caído la educación física al ser considerada como un saber confluente de conocimientos provenientes de las ciencias naturales y sociales, que llegan y se alejan sin afectarse en sí mismos y sin permitir desde ellos la configuración de un cuerpo de conocimiento nuevo y con estatuto académico propio.

$\mathrm{Al}$ asumir la construcción de lo humano desde la acción motriz, en la dialéctica corporalidad-motricidadcontexto, desde la cual, como síntesis, se logra un nivel superior humano y de humanización, bien podría plantearse aquello que Teilhard de Chardin llamó noosfera: la creación de una nueva armonía entre los humanos,

1261 en la que técnica y poesía, producción y espiritualidad, corazón y pensamiento encuentran una sintonía más alta y sinfónica, y donde la educación física se constituye en la disciplina encargada de construir esa noosfera conceptual -si es que el término lo permite-, la cual sólo tendría razón de ser en el contexto de la cotidianidad de la vida humana.

En este sentido, es necesario construir la disciplina de la educación física orientada hacia el desarrollo del potencial humano, multidimensional y unitaria, en el contexto específico de sus relaciones, o como lo plantea José María Cagigal, al englobarla en tres grandes metas: "1. El hombre dueño de sí mismo; 2. El hombre en el espacio, y 3. El hombre en el mundo social" (Cagigal, citado en Trigo, 2000).
Psicomotricidad:

alternativa para el desarrollo humano desde la educación física

En este aparte, el propósito consiste en mostrar "la psi comotricidad como alternativa de reivindicación de la importancia del cuerpo y el movimiento para el desarrollo humano", describiendo que la estructuración de lo humano se logró desde el movimiento, y señalando cómo el paso por tres componentes de modelos organizados de movimiento (el postural, el de transporte y el de la manipulación), provocó la conformación de la corteza cerebral (cerebración), y con su aparición, en primera instancia, se facilita el refinamiento superior de lo motor $y$, en un segundo momento, el desarrollo de lo simbólico, con el logro del lenguaje y el pensamiento.

La intención es consolidar un reconocimiento de lo motor, como la base del desarrollo de lo humano hast llegar a la conformación del intelecto, teniendo como base la construcción de "corteza cerebral", desarrollada en el proceso evolutivo para cualificar lo motor, tratando de superar la infravaloración del intelecto, cuando desde el proceso histórico de la evolución del hombre, la conformación de la corteza cerebral se manifestó como alternativa de facilitación de las posibilidades motoras de adaptación, fundamentales para la supervivencia (Pachón, 2005: 4).

Se resalta la importancia del movimiento como el motor dinámico de lo cultural, no sólo en la elaboración de elementos tecnológicos, sino en lo más trascendente; es decir, el alcance de la subjetividad humana, que tiene su comienzo con el reconocimiento del otro, su confrontación con lo moral, hasta llegar a la abstracción y la simbolización desde el lenguaje y el pensamiento.

Hay que resaltar también la conquista de la posición bípeda, que ocasiona la liberación de las manos para que, actuando en correlación con los ojos, den inicio a la integración perceptivo-motora, desde la cual, y a partir de las sensaciones y percepciones, se siga a las representaciones, los conceptos y, desde luego, al pensamiento (Pachón, 2005: 4).

La evolución humana ha dejado de ser sólo biológica, para convertirse centralmente en social y cultural, y representada en el desarrollo de la ciencia y la tecnología, la ética, estética, la lúdica y demás categorías que constituyen lo significativamente humano, y que apenas se encuentra en el comienzo de su nivel evolutivo.

Por eso, se plantea "la psicomotricidad como alternativa de reivindicación de la importancia del cuerpo y el movimiento para el desarrollo humano", con la inten- 
ción de abrir un nuevo horizonte para la educación físi$c a$, dándole reconocimiento a la importancia del cuerpo en la búsqueda del desarrollo humano, para superar la supremacía instrumental hacia la tecnificación deportiva y la competitividad en que se encuentra inmersa, y brindándole, además, una alternativa más humana, al comprender que lo motor sigue siendo esencial en la educación y formación de lo humano, teniendo en cuenta que la filogenia se replica en la ontogenia ( $\mathrm{Pa}$ chón, 2005: 5).

No obstante, queda el interrogante de cómo generar al cuerpo desde la educación física, la importancia que le corresponde, para que de allí se parta hacia un desarrollo fundamental de la subjetividad humana, a través de la psicomotricidad como alternativa de reivindicación de la trascendencia del cuerpo y el movimiento para el desarrollo humano, que facilita los medios, espacios y alternativas para que la educación física pueda preparar líderes como protagonistas cualificados, que logren sacarla de su deterioro actual.

En consecuencia, es necesario elaborar, plantear y argumentar estrategias desde la psicomotricidad, para que consoliden el reconocimiento de la educación física como la alternativa para rescatar la importancia del cuerpo y la acción motriz en el proceso educativo, y desde lo motor pueda estructurarse toda la realidad multidimensional y unitaria, hasta la subjetividad que implica el pensamiento formal, lo estético, lo ético, lo lúdico y la interacción con otros.

No obstante, quedan cuestionamientos e interrogantes como los siguientes:

- ¿Cuáles son las características generales de la psicomotricidad y de la educación psicomotriz?

- ¿Cómo generar al cuerpo desde la psicomotricidad la importancia que le corresponde?

- ¿Cuáles son las características fundamentales de un planteamiento educativo que rescate la importancia del cuerpo y el movimiento como factores esenciales del desarrollo humano?

- ¿Cuáles son las particularidades de una propuesta que redimensione, contraste y dinamice el significado de la psicomotricidad y su influencia en el desarrollo de lo humano?

\section{Qué se entiende por psicomotricidad}

"La Psicomotricidad nace como un esfuerzo para superar la concepción dualista cartesiana del hombre con el fin de recomponer y respetar su unidad" (Boscaini, 2002, itáli- cas fuera del original). Un individuo debe considerarse dentro de un contexto íntegro y global, que surge como resultado de distintos procesos que se dan durante su evolución.

Cuando hacemos referencia a la parte motriz, biológica, tratamos con el proceso de maduración orgánica del cuerpo físico, su estructura y su funcionamiento. Además, está la parte ambiental, que señala el medio donde se desenvuelve la persona y las distintas relaciones que pueden darse dentro de él. Ambos procesos dependen uno del otro y se dan a la vez, no en paralelo, sino en conjunta armonía.

Así, un desorden a nivel orgánico tiene también sus consecuencias a nivel relacional, y viceversa. El síntoma no es local. No puede tratarse con él de manera aislada. La psicomotricidad no trata la mente y el cuerpo por separado. Entiende al individuo como una esfera que los engloba de manera dinámica y armoniosa en su constante relación con el medio.

Al tratar con la psicomotricidad, es común encontrar que existe un proceso de maduración neurológica que le asegura a la especie humana crear una entidad interna que le permita al mismo tiempo percibir y percibirse. Vitor Da Fonseca (1998) sugiere un sistema psicomotor humano en el que, de manera escalonada y con base en siete factores (conductas neuromotrices y perceptivomotrices), el individuo logra el dominio físico yendo "del acto al pensamiento" (Berruelo, 2002).

Sin embargo, este planteamiento se queda un poco corto, porque nos referimos al campo de acción de la psicomotricidad y su práctica educativa (o reeducativa o clínica), pues omite grandes aspectos, entre ellos los corporales, como la expresión, la relación y la emoción. A partir de ello, a continuación expondremos el sistema psicomotor humano señalado por Vitor Da Fonseca (1998) y la manera en que se asocia cada factor con los demás, traduciéndose en capacidades únicas de la especie.

\section{El sistema psicomotor humano}

Nacemos con una relación inconclusa entre el cuerpo y el cerebro que no tiene vías de comunicación, ni de interacción. Es el desarrollo de la motricidad lo que proporciona esa comunicación estrecha entre el centro y la periferia. Una vez conquistada esa interacción integral del cuerpo y del cerebro surgen nuevas propiedades, nuevas capacidades, nuevas funciones de aprendizaje y de relación con el otro.

Esas nuevas capacidades y esas nuevas funciones de aprendizaje surgen en la especie humana como la cuali- 
dad llamada psicomotricidad. Para poder ocuparse con las funciones psíquicas más elevadas, como la lectoescritura, o sea, el trabajo con símbolos, el cerebro humano tiene que organizar primero la información venida de su cuerpo. Así, procesar la información venida del exterior requiere una atención especial, sólo posible una vez que el interior ha sido regulado.

No es posible empezar al contrario. Siempre es necesario comenzar por lo propio, por lo que está cerca, para entonces poder avanzar. Pero si lo básico no es manejado, aquello que requiera algo de complejidad nunca podrá ser reconocido.

De este modo, el aprendizaje es sinónimo de integración sensorial. El cuerpo se organiza motora y psíquicamente por medio de siete factores (tonicidad, equilibrio, lateralidad, noción del cuerpo, estructuración espacio-temporal, motricidad gruesa y motricidad fina) que comprenden su dominio interno y el dominio del exterior.

Esta cualidad, inherente a la especie humana, se denomina psicomotricidad porque comprende el camino del dominio físico hasta la creación de un "yo" interno (lo psíquico) que posteriormente guiará el movimiento a voluntad. Aun así, sin el dominio físico, es imposible prestar atención a estímulos exteriores.

Los siete factores anteriormente nombrados se distribuyen en tres unidades funcionales básicas, de las cuales se compone el cerebro. Cada una de ellas posee cualidades y características propias que le permitirán realizar las tareas a las cuales están asociadas. Las tres unidades se organizan estructuralmente, de manera que su trabajo conjunto traduce el potencial mental humano, desde el movimiento voluntario hasta la creación del lenguaje. Éstas son:

- Primera unidad funcional, para regular el tono cortical y la función de vigilancia.

- Segunda unidad fundamental, para obtener, captar, procesar y almacenar información venida del mundo exterior.

- Tercera unidad fundamental, para programar, regular y verificar la actividad mental (Boscaini, 2002).

La tonicidad y el equilibrio se ubican en la primera unidad. La lateralidad, la noción del cuerpo y la estructuración espacio-temporal en la segunda y finalmente, la motricidad fina y la gruesa en la tercera.

La psicomotricidad, cualidad única en la especie humana, es, en resumen, la integración de la información propioceptiva (datos tónicos, kinestésicos, vestibulares y motores) con la información exteroceptiva (datos visuales, auditivos, espaciales, temporales) lo cual origina niveles psíquicos más complejos. La motricidad se presenta en el cuerpo humano para dar origen a la psicomotricidad y someterse después a los designios psíquicos. El movimiento crea la voluntad para después ser dirigido por ella, expresando a través del cuerpo su propio lenguaje, cargado de emoción y vida.

\section{Una aproximación \\ a la noción de cuerpo}

Desde la lateralidad y hasta la estructuración espacio temporal -la segunda unidad fundamental del cerebro humano-, se establece un puente entre lo meramente físico, la adquisición del tono y el dominio de la bipedestación, hasta lo intangible en el cuerpo humano; es decir, la noción de un yo interno. La lateralidad integra perfectamente ambas cualidades. La información propioceptiva y la exteroceptiva, una vez integradas y analizadas, se almacenan y sintetizan en forma de posturas corporales y modelos de movimiento, cuyas referencias, a partir de la experiencia corporal, integran lo emocional. La cualidad subjetiva aparece en el niño para más tarde, a través del movimiento, expresar el sentimiento.

El cuerpo, convencido físicamente y referenciado internamente, se torna un instrumento del pensamiento, a través del cual éste se comunica. La constitución del yo interno, el ego, la noción del cuerpo, es de gran significado en los procesos de aprendizaje, ya que instituye la personalidad y la motricidad psicológica de las que surgen todo tipo de comportamientos y actitudes.

"A lo largo de la experiencia, el cerebro organiza una imagen sensorial interna a partir de la actividad motora, imagen que se refina y hace más precisa, se estructura y actualiza, se almacena y se utiliza" (Da Fonseca, 1998: 193). Como fundamento de la personalidad y sustento de los comportamientos, la imagen interna que el niño tiene de sí mismo es la base de cualquier movimiento a voluntad. La noción del cuerpo se convierte en un mapa de orientación del espacio exterior.

Una vez interiorizada, creada la imagen del propio cuerpo, ésta se utiliza como referencia hacia los objetos. Algo es pesado o liviano, grande o pequeño, está cerca o lejos, con relación al propio cuerpo. Y sus implicaciones en el desarrollo cognitivo son claras.

\section{La dimensión del movimiento humano}

Tal vez es difícil para los educadores entender que no hay momentos para el cuerpo y momentos para 
el intelecto. Quizá si se aceptara que el intelecto es cuerpo, que el cuerpo es sensibilidad, que la imaginación es la puerta a la ciencia, las escuelas serían más divertidas y los niños tendrían menos dificultad en concentrarse. De igual manera, con mayor o menor libertad, un largo período de la vida se ocupa en entrenar el cuerpo para que su eficacia no sea traicionada por la maravillosa capacidad de sus sentidos (Cajiao, 1996: 128).

El cuerpo es el lugar donde se escribe la historia. El grado de maduración cronológica, mental y emocional de una persona puede verse grabado en el cuerpo. Así, las posturas y el movimiento (que es una secuencia intermitente de posturas), cobran gran importancia en la psicomotricidad. Al movimiento se le atribuye más que un estado funcional que expresa el aspecto fisiológico meramente orgánico. El movimiento es también un medio de expresión en que el cuerpo se utiliza para "hablar". Se le atribuye a éste un sentido comunicativo, afectivo y emocional que evidencia el sentido de lo humano.

El movimiento de cada personaje se acondiciona a las adaptaciones y ajustes que éste debe realizar en la búsqueda de sortear correctamente las necesidades impuestas por el medio. Por eso, cada movimiento es un sello personal aunque todos partamos del mismo punto y presentemos las mismas capacidades motoras y adaptativas. La forma en que cada uno entiende el mundo y expresa su manera estar-dentro-de-él, torna peculiar e movimiento; como si habláramos de la firma o la hue lla digital. La manera en que caminamos, cumplimos, saludamos, dormimos, etc., indica rasgos de nuestra personalidad y nuestro estado de ánimo, que permiten realizar innumerables lecturas del cuerpo humano expresándose a través de los gestos.

Existen varios vocablos referidos específicamente a movimiento, que me gustaría tratar ahora, según la descripción hecha por Pedro Berruelo (2002). El autor cit los términos: movimiento, reflejo, voluntario, natural, espontáneo, artificial, automático, expresivo y funcional. El movimiento humano, traducido en acción, necesita de elementos como la coordinación de diferentes grupos musculares y la intención de llevarlo a cabo. A esta acción o conducta motriz se le conoce como praxia. Uno de los significados más relevantes que adquieren las praxias, dentro del movimiento humano, es su carácter expresivo, por lo que incide en el desarrollo de pensamiento y del lenguaje.

Jean Le Bouch, citado por Pedro Berruelo (2002), distingue tres tipos de praxias, según su intención: las transitivas, las simbólicas y las estéticas. Las praxias transitivas indican un deseo de modificar el objeto sobre el cual se centra la atención. Las simbólicas, denotan un deseo de comunicar algo mediante el gesto. Y, las estéticas, que también pretenden comunicar una idea o un sentimiento, dan mayor prioridad al medio que utiliza para hacerlo que a la precisión misma del mensaje.

No resulta difícil ahora entender por qué la psicomotricidad estudia el movimiento como una producción corporal de un individuo que lo utiliza para relacionarse con el otro, no sólo de manera funcional, sino también comunicativa y emocional.

El arte, la más noble y profunda evidencia de que el interior de una persona se hace tangible a través de su cuerpo, invita, de manera silenciosa y apasionante, a crear un lenguaje donde las palabras se tornen colores sobre el lienzo, notas sobre las cuerdas y armoniosos desequilibrios de las posturas. Un lenguaje donde, una vez conocido lo que puede hacer el cuerpo, las maravi llosas capacidades de sus sentidos se invierten para en tonces mirar con el tacto, tocar con los ojos y hablar con el cuerpo. Y ese sentido de lo humano, me parece, debe ser el sentido de la educación: mostrar el camino por donde la persona pueda encontrar su propia manera de "moverse" en el mundo.

\section{Los espacios académicos de acción,} recursos y método de intervención de la educación psicomotriz

La educación psicomotriz es un campo bastante amplio, y no se trata únicamente de un tipo de educación, porque, si bien es cierto que guarda sus diferencias con la educación tradicional y las técnicas de intervención en reeducación psicomotriz, esto no significa que no esté directamente relacionada con ellas. Existe, por fortuna, este campo de acción que se convierte en salón escolar y la sala de terapia en una sala de psicomotricidad, y viceversa.

Las diferencias que caracterizan estas tres formas de educación radican en la manera en que entienden y se relacionan con el infante y el niño, y las técnicas que utilizan para tal fin. Sin embargo, una vez entendidas bajo el manto del individuo y del grupo en que se desenvuelve, su intervención las presenta casi como una sola. Según Josefina Sánchez (2002: 111):

la educación psicomotriz se lleva a la práctica mediante una pedagogía del descubrimiento, a partir de la exploración de situaciones espontáneas variadas que van a llevar al educando, según sus características personales y sus vivencias, a procesos de relación y maduración diferentes. 
En todo caso, para que cada proceso de relación y cada vivencia puedan realmente llegar a ser explotados de manera positiva hacia la maduración del niño y de la niña, los profesores deben basar la estructuración de los programas y su modificación según la respuesta del in fante, en los intereses del grupo; es decir, en los intereses de los alumnos, sus circunstancias, diferencias y deseos para lograr, entonces, que la educación se adapte a los estudiantes y no que éstos moldeen su desarrollo hacia cánones ya preestablecidos.

De tal manera que, en la educación especial, o reeducación -según el punto de vista que se está tratando ahora, aunque sería igual de válido para cualquier otro tipo de educación-, la intervención es guiada por la necesidad de encontrar nuevos recursos y métodos para empezar desde las potencialidades del niño, hecho que genera un respeto hacia la expresividad, y no desde sus posibles problemas de desarrollo, pues, de lo contrario, se pretendería que el estudiante aprendiera según los intereses y objetivos del profesor y la creación del programa se vería canalizada únicamente hacia la superación de un déficit psicomotor. Es imprescindible que el psicomotrista se abra a los diferentes ritmos y expresiones individuales de cada uno de sus alumnos (por eso, los grupos deben ser pequeños; en lo posible, no más de diez niños), desarrollando su escucha corporal hacia lo que cada uno de ellos es.

\section{Conclusiones}

Vimos cómo el cerebro humano va adquiriendo complejidad durante su maduración neurológica, pasando del movimiento reflejo al voluntario, creando la noción del yo, que, en sí misma, constiture lo psíquico. A continuación, expondremos las características de la educa-

130| ción psicomotriz y su significado en la edad infantil.

Es importante, al tratar con la educación psicomotriz, definir, en primer lugar, lo que se entiende por educación y cuál es su relación específica con la psicomotricidad. Existen variadas clasificaciones de educación, según su concepción, que se diferencian por su función o finalidad. Zabalza, citado por Josefina Sánchez (2002: 98) clasifica la educación en cinco concepciones sociales: como desarrollo, como liberación, como capacitación socio-laboral, como información y como formación.

En este caso en particular, y siempre que se hable de educación psicomotriz, la orientación se dará hacia la globalidad del ser (parte psíquica y parte motriz) y por eso consideramos más acertadas las concepciones de desarrollo, formación y liberación, según la clasificación de Zabalza (1990).

Con base en estas tres últimas concepciones, el objetivo general de la educación sería el de "facilitar en cada sujeto la construcción consciente de sus formas de pensar, sentir y actuar, mediante el ejercicio libre de su independencia intelectual" (Zabalza, citado en Sánchez, 2002), y bajo esta perspectiva no hay mucha diferencia entre el objetivo que persigue la educación "normal", por así llamarla, y el de la práctica psicomotriz. Sin embargo, ha de tenerse presente que la intervención psicomotriz encaminará su atención a lo corporal, mejorando el ajuste del sujeto a su entorno, equilibrando, como diría Josefina Sánchez, su independencia intelectual (2002); es decir, su independencia corporal y emocional.

La educación es un proceso de descubrimiento, relacionamiento y superación. Pero no podemos relacionarnos con algo en la medida en que nuestra atención se centra en lo que vamos a conseguir. Por eso decimo que el niño debe descubrir y superar su propio medio a través del juego. Porque en el juego no hay futuro ni pasado, sólo presente, sólo atención al momento. De esa manera, se avanza al ritmo apropiado. Si se presta atención al presente, el movimiento y el aprendizaje surgen en el niño como necesidad, como expresión y no como asimilación de unas formas que le son ajenas.

La educación psicomotriz, en efecto, tiene un gran énfasis en el movimiento coordinativo, pero no es en la asimilación del gesto en lo que se basa. Estas pruebas pretenden no otra cosa que proporcionar al niño la mayor cantidad posible de experiencias en búsqueda del enriquecimiento y en desenvolvimiento corporal y actitudinal. En otras palabras, busca enriquecer su exploración sensorial del medio y con el medio. Así, "hay más posibilidad de aprender cuantos más elementos de medio sean incorporados en el proceso de retroalimentación" (Gutiérrez Delgado, s. f.). Cuanto más interesado se muestre el niño, tanta más oportunidad habrá de introducir experiencias que lo enriquezcan.

La intervención psicomotriz es una práctica afectiva donde existe un diálogo tónico, hay un encuentro corporal entre el niño y el psicomotrista, que anula la relación visoauditiva de la escuela normal. El psicomotrista percibe al niño, lo siente, pero no se transforma en él. Lo ayuda a madurar mediante la relación afectiva (que le da sentido a lo humano) que surge entre los dos.

El psicomotrista escucha al niño cuando lo obsèrva y dispone su cuerpo hacia un diálogo tónico. Observar al niño quiere decir conocerlo, hablarle mediante la mirada, estar presente en sus producciones y enseñarle a 
valerse de sus ojos, de su cuerpo y de su movimiento. El contacto visual es muy importante; indica que mi atención está con él, que lo sigo y lo cuido. Necesito mirarlo, si quiero, mediante la relación afectiva que entablamos, mostrarle y hacerle entender lo que puede el cuerpo.

Finalmente, en cumplimiento del propósito de presentar "la psicomotricidad como alternativa de reivindicación del cuerpo y el movimiento para el desarrollo humano", a partir de que uno de los problemas fundamentales de la educación física está centrado en

\section{Referencias}

Barrow M., Harold y Brown, J. (1992). Hombre y movimiento. Barcelona: Ediciones Doyma.

Berruelo, P. (2002). Las conductas motrices. En: La práctica psicomotriz. Málaga: Ediciones Aljibe.

Boscaini, F. (2002). Nuevas necesidades y nuevas respuestas. En: La práctica psicomotriz, Málaga: Ediciones Aljibe.

Cagigal, J. M. (1979). Cultura intelectual y cultura física. Buenos Aires: Editorial Kapelusz.

Cajiao, F. (1996). La piel del alma. Bogotá: Cooperativa Editorial Magisterio.

Da Fonseca, V. (1998). Manual de observación psicomotriz. Barcelona: Inde.

De la Guardia, R. M. (2002). La formación del psicomotricista. Las relaciones familia-profesional. En: La práctica psicomotriz. Málaga: Ediciones Aljibe.

De Zubiría, S. J. y De Zubiría, M. (1997). Fundamentos de pedagogía conceptual. Bogotá: Plaza \& Janes.

Flórez Ochoa, R. (1997). Hacia una pedagogía del conocimiento. Bogotá: D’Vinni.

Gardner, H. (1995). Estructuras de la mente. La teoría de las inteligencias múltiples. México: Fondo de Cultura Económica.

González, A. (2002). La práctica psicomotriz de B. Aucouturier. En: La práctica psicomotriz, Málaga: Ediciones Aljibe.

Grupe, O. (1976). Teoría antropológica de la Educación Física. Madrid: INEF.

Gutiérrez Delgado, M. (s. f.) 140 juegos de educación psicomotriz. Editorial Deportiva. 3a. edición. 3a. ed. s. l. el dualismo mente-cuerpo, a través del desarrollo argumentativo de este documento se ha pretendido mostrar que cerebro y cuerpo no son dos esferas diferentes, sino una sola, interdependiente y organizada. El cerebro es el órgano de la motricidad y el cuerpo es la base sobre la cual se edifica lo psíquico. Si no tuviéramos cuerpo, simplemente no podríamos pensar, y nuestra condición de seres conscientes con capacidad de aprendizajes superiores se da debido a la estructuración de nuestro cerebro a través del movimiento físico.

Le Bouch, J. (2001). El cuerpo en la escuela del siglo XXI. Barcelona: Inde.

1995. El desarrollo psicomotor, desde el nacimiento hasta los seis años. Barcelona: Paidós Ibérica.

Llorca, M., Ramos, V., Sánchez, J. y Vega, A. (2002). La práctica psicomotriz. Málaga: Ediciones Aljibe.

Meinel, K. (1977). Didáctica del movimiento. La Habana: Editorial Orbe.

Navarro, A. (2002). El trabajo psicomotriz en la escuela infantil. En: La práctica psicomotriz. Málaga: Ediciones Aljibe.

Pachón, O. (2005). Educación física y desarrollo humano. En: Kinesis. Revista de Ciencias del Deporte, Educación Física y Recreación, 41 , p. 9.

Ribeiro Da Silva, A. (1975). Psicología del deporte y preparación del deportista. Buenos Aires: Editorial Kapelusz.

Rodríguez López, J. (1998). Deporte y ciencia. Barcelona: Inde.

Sánchez, J. (2002). La educación psicomotriz en el contexto de las ciencias de la educación. En: La práctica psicomotriz, Málaga: Ediciones Aljibe.

Trigo, E. (2000). Fundamentos de la motricidad. Madrid: Editorial Gymnos.

Zemelman, H. (2004-2005). "Desafíos en el proceso de formación docente que plantea el actual contexto histórico latinoamericano". conferencia posdoctoral. Bogotá, 1 de junio-2 de diciembre. 